\title{
Factors associated with knowledge level in adult type 1 diabetic patients
}

\section{ABSTRACT}

Background: The objective of the study is to determine the factors associated with the level of knowledge of Tunisian type 1 diabetic (T1D) patients in adulthood. Methods: This is a cross-sectional study including 93 T1D patients over 18 years old. The knowledge assessment was carried out by a questionnaire rated out of 20 points. The subjects with an "unsatisfactory" level of knowledge (score < 10/20) were compared with subjects whose level of knowledge was "satisfactory" according to their socio-demographic, clinical, and paraclinical characteristics.

Results: The mean age of the patients was $37.2 \pm$ \pm 12.4 years. The level of knowledge was "unsatisfactory" in 21 patients (23\%). After univariate analysis, an "unsatisfactory" level of knowledge was associated with a low level of education ( $p=0.001)$, a poor socioeconomic level ( $p=0.03)$, a poor glycemic control ( $p=0.003$ ) and the absence of self-monitoring ( $p=0.002$ ). After multivariate analysis, only a low level of education and a lack of practice of self-monitoring were associated with an "unsatisfactory" level of knowledge (respectively $p=0.03$ and 0.03 ; adjusted OR $[95 \%$ confidence interval] $=7.3[1.2-43.5]$ and 13.7 [1.3-143.3]).

Conclusions: The factors independently associated with the level of knowledge in adult T1D patients are the level of education and the practice of self-monitoring.

Address for correspondence:

Meriem Yazidi

Department of Endocrinology, University of Tunis El Manar

Faculty of Medicine of Tunis, La Rabta Hospital

1007 Tunis, Tunisia

e-mail: meriemyazidi@gmail.com

Clinical Diabetology 2022, 11; 1: 1-5

DOI: 10.5603/DK.a2022.0001

Received: 29.01.2021

Accepted: 3.042021
This encourages better tailoring of educational messages to patients with low levels of education and suggests that a better level of knowledge ensures better self-management of diabetes. However, the relationship with the quality of glycemic control remains uncertain. (Clin Diabetol 2022, 11; 1: 1-5)

Keywords: type 1 diabetes, knowledge, therapeutic education, adult

\section{Introduction}

Acquiring knowledge about the disease and its treatment is essential to improve glycemic control and quality of life of type 1 diabetic patients (T1D). The majority of studies that have focused on the assessment of knowledge and the level of therapeutic education in T1D patients often only concerned children and adolescents [1-4]. Few studies have assessed the level of knowledge of T1D patients in adulthood. Studies in adult patients often did not differentiate between T1D and type 2 diabetic patients [5-7]. In addition, the link between the level of knowledge and the quality of glycemic control in diabetics is very controversial $[4,5$, 8-11]. The aims of this study were to assess the level of knowledge of a Tunisian population with type 1 diabetes in adulthood and to identify the socio-demographic, clinical, and paraclinical factors associated with it.

\section{Methods}

\section{Study population}

This is a cross-sectional study carried out in 93 T1D patients followed in the endocrinology department of La Rabta hospital in Tunis. The inclusion criteria were age greater than or equal to 18 years and a duration of disease $>1$ year. Pregnant women were not included in the study. 
The diagnosis of type 1 diabetes was made if signs of absolute insulin deficiency (weight loss, ketoacidosis) were present in a subject under the age of 30 or in the presence of positive anti-glutamic acid decarboxylase 65 antibodies (anti GAD65) or islet cell antibodies (ICA) antibodies.

In our teaching hospital, therapeutic education is systematically provided by the care team during hospitalizations of patients. Additional education sessions in the day hospital are carried out during the followup if the attending physician deems it necessary. The education program is standardized and covers the mechanisms of diabetes, complications of diabetes, dietetics, pharmacological treatment of diabetes, glycemic self-monitoring, and glycemic targets.

\section{Study protocol}

Socio-demographic parameters, the duration of diabetes, and current treatment have been collected.

A physical examination (weight, height, body mass index, lipodystrophies) was performed.

The socioeconomic level was estimated based on the type of social coverage. Patients receiving a reduced-rate care card or a free care card were classified as having poor socio-economic status.

Patients who were illiterate or had a primary education level were classified as having a low level of education.

The mean of glycated hemoglobin (HbA1c) levels of the last year was calculated and used as a glycemic control indicator. The HbA1c was measured by highperformance liquid chromatography (HPLC) at the biochemistry department of the Rabta hospital. The glycemic control was considered poor if the mean of $\mathrm{HbA} 1 \mathrm{c}$ of the last year was higher than $8 \%$.

The level of knowledge was assessed using a questionnaire comprising 20 questions in the Tunisian dialect. The questionnaire was read by the same operating physician, in a neutral and non-suggestive manner. Each patient was informed in advance of our intention to assess their knowledge of the disease and its management.

The areas of knowledge tested were:

- general knowledge of the mechanism of type 1 diabetes, acute metabolic complications (ketoacidosis), and chronic complications of diabetes;

- the insulin injection technique: times, sites, lipodystrophies;

- hypoglycemia: signs, threshold value, action to take and causes;

- hyperglycemia: signs and causes;

- glycemic self-monitoring and glycemic targets: frequency of blood glucose monitoring, fasting and postprandial blood glucose targets, the meaning of glycated hemoglobin and its target value;

- certain specific situations: infection, digestive disorders, and physical activity.

A score ranging from 0 to 1 point was assigned to each question depending on whether the answer was correct or not. A score out of 20 points was assigned to each patient at the end of the questionnaire. The level of knowledge was classified as "unsatisfactory" if the score obtained in response to the questionnaire was $<10 / 20$ and "satisfactory" otherwise. The level of knowledge was considered "high" if the score obtained was > 15/20 and "medium" if the score obtained was between 10 and 15/20.

The study was approved by the hospital's medical ethics committee.

\section{Reliability and validity of the questionnaire}

The reliability of the questionnaire was verified by applying the internal consistency formula, which checks whether the different items of the questionnaire explore the same performance domain allowing the calculation of the Cronbach $\alpha$ score, which is considered satisfactory if it is higher than 0.7.

The validity of the content of the questionnaire was estimated by applying the Kolmogorov-Smirnov test which verifies the distribution of scores obtained according to the normal distribution.

\section{Statistical analysis}

Data entry and analysis was performed using SPSS software version 20.0. Quantitative variables were expressed as mean \pm standard deviation and qualitative variables were expressed as percentage (\%). In order to identify the factors associated with the level of knowledge of T1D patients, we subdivided the patients into two groups according to their level of knowledge ("sat isfactory"/"unsatisfactory"). Pearson's Chi-squared and Fisher's exact tests were used to compare proportions In order to identify the risk factors independently related to the level of knowledge of T1D, we conducted a multivariate binary logistic regression analysis including all the factors whose " $p$ " were $<0.15$ on univariate analysis. In all statistical tests, the significance level was set at 0.05 .

\section{Results}

The Cronbach $\alpha$ score, assessing the internal consistency of the questionnaire, was 0.77 , therefore satisfactory. Application of the Kolmogorov-Smirnov test confirmed the Gaussian distribution of the responses obtained.

The mean age of the patients was $37.2 \pm 12.4$ years (19-76), 46\% were men and 54\% were women. The mean duration of diabetes was $12.0 \pm 8.6$ years. 
Table 1. Comparison of clinical and biological parameters of type 1 diabetic patients according to their level of knowledge

\begin{tabular}{lcccc}
\hline Parameter & $\begin{array}{c}\text { "Unsatisfactory" level of } \\
\text { knowledge } \\
(\mathbf{n}=\mathbf{2 1})\end{array}$ & $\begin{array}{c}\text { "Satisfactory" level of } \\
\text { knowledge } \\
(\mathbf{n}=\mathbf{7 2})\end{array}$ & OR (95\% Cl) \\
\hline Age > 40 years (\%) & 52 & 32 & 0.09 & $2.3(0.8-6.1)$ \\
Female gender (\%) & 67 & 50 & 0.17 & $2.0(0.7-5.5)$ \\
Married (\%) & 50 & 51 & 1.0 & $1.0(0.1-2.0)$ \\
Diabetes duration > 10 years (\%) & 43 & 44 & 0,94 & $0.9(0.4-2.6)$ \\
Poor socio-economic status (\%) & 40 & 16 & 0.03 & $3.4(1.1-10.4)$ \\
Low level of education (\%) & 71 & 30 & 0.001 & $5.7(1.9-16.6)$ \\
Absence of self-monitoring (\%) & 86 & 47 & 0.002 & $6.7(1.8-24.7)$ \\
Hospitalization for ketoacidosis (\%) & 10 & 10 & 0.97 & $0.9(0.2-5.1)$ \\
Hypoglycemic events (\%) & 76 & 82 & 0.54 & $0.7(0.2-2.3)$ \\
Lipodystrophies (\%) & 22 & 25 & 1.0 & $0.8(0.2-2.9)$ \\
Diabetic retinopathy (\%) & 17 & 20 & 1.0 & $0.8(1.2-3.3)$ \\
Diabetic nephropathy (\%) & 18 & 26 & 0.54 & $0.6(0.1-2.4)$ \\
Diabetic neuropathy (\%) & 50 & 31 & 0.17 & $2.1(0.7-6.9)$ \\
Poor glycemic control (\%) & 93 & 51 & 0.003 & $13.5(1.7-109.6)$ \\
\hline
\end{tabular}

$\mathrm{Cl}$ - confidence interval; OR — odds ratio

The socioeconomic level was poor in $22 \%$ of patients and educational level was low in $40 \%$ of patients. The mean $\mathrm{HbA} 1 \mathrm{c}$ level in the last year of follow-up was $8.4 \pm 1.4 \%$ (5.5-11.9).

The mean score obtained in response to the questionnaire was $12.8 \pm 3.3 / 20$ (4-18). The proportion of correct answers was $64 \%$. The level of knowledge was "unsatisfactory" in 21 patients (23\%), "medium" in 41 patients (44\%), and "high" in 31 patients (33\%). The areas of knowledge with the lowest rates of correct answers $(<50 \%)$ concerned the mechanism of type 1 diabetes, the significance and target value of $\mathrm{HbA1C}$, the significance of acetonuria, the management of hypoglycemic events, insulin dose adjustment for exercise and sick day management.

The study of the relationship between the various clinical and biological parameters and the level of knowledge of the patients is shown in Table 1. Table 2 shows the results of the multivariate analysis.

\section{Discussion}

The therapeutic education of diabetic patients in Tunisia is confronted, as in many other developing countries, with several difficulties linked mainly to the low level of education and the low socioeconomic status of a large proportion of the population. The level of knowledge of adult T1D patients about their disease and its management was medium in this study. The level was unsatisfactory in almost a quarter of the patients. Factors independently associated with an un-
Table 2. Factors associated with an "unsatisfactory" level of knowledge after multivariate analysis

\begin{tabular}{lcc}
\hline Parameter & $\mathbf{P}$ & $\begin{array}{c}\text { Adjusted OR } \\
(95 \% \mathrm{CI})\end{array}$ \\
\hline Age > 40 years & 0.46 & $0.5(0.1-2.9)$ \\
Poor socio-economic status & 0.57 & $1.5(0.3-7.3)$ \\
Low level of education & $\mathbf{0 . 0 3}$ & $7.3(1.2-43.5)$ \\
Absence of self-monitoring & $\mathbf{0 . 0 3}$ & $13.7(1.3-143.3)$ \\
Poor glycemic control & 0.09 & $6.9(0.7-66.5)$ \\
\hline
\end{tabular}

$\mathrm{Cl}$ - confidence interval; OR — odds ratio

satisfactory level of knowledge were a low level of education and a lack of self-monitoring of blood glucose.

Our study has some limitations. Indeed, the study subjects were all recruited from a public health structure. Those patients often have a low socioeconomic and educational level. In addition, some areas of knowledge were not assessed by the questionnaire. The assessment of dietetic knowledge was not addressed. The assessment of physical activity goals and knowledge about preventing foot injuries were also overlooked.

It's difficult to compare the different studies evaluating the knowledge of diabetic patients given the heterogeneity of the study populations. Most often, the level of knowledge of T1D patients is higher than that of type 2 diabetic patients [12-14].

The evaluation of the level of knowledge by domain revealed that the topics with the lowest proportions of 
correct answers mainly concerned the theoretical aspect of the disease, such as the mechanism of diabetes or the significance of $\mathrm{HbA} 1 \mathrm{c}$, while the aspects relating to the day-to-day management of the disease, such as the modalities of insulin injection, hypoglycemic and hyperglycemic events, were better known by the patients.

This finding can be explained by the fact that the level of education was one of the main factors associated with the level of knowledge of the patients and that $40 \%$ of the patients had a low level of education in our study. The relationship between the level of knowledge and the level of education has been demonstrated by several other studies assessing the knowledge of diabetics [7, 8, 15-17]. This association is explained by the greater ability of highly educated subjects to understand and use information from healthcare professionals [9]. The motivation and the ability to access and use the information are largely linked to the intellectual and social achievements of each individual. This significant association also demonstrates that the information provided by healthcare professionals is less suitable for patients with low levels of education. These patients need visual and auditory messages more than written messages and more demonstrations than theoretical information [18-19].

The second factor associated with the level of knowledge of patients was whether or not self-monitoring was practiced. Indeed, the lack of practice of selfmonitoring was independently associated with a lower level of knowledge in this study. Thus, a better level of knowledge would ensure better self-management of diabetes. However, some authors demonstrated the absence of a relationship between knowledge and the practical aspect of management of the disease ("Knowledge-behavior gap") [7, 13, 20, 21]. The link between knowledge and practice depends on several factors related to the patient, his immediate environment, health professionals, and the care structure.

The association between the level of knowledge of diabetics and the quality of glycemic control is, however, very controversial. The results of studies that have looked into this subject are very heterogeneous [4, 7, 8-11]. In our study, a lower level of knowledge was associated with poor glycemic control. However, this association was no longer significant after controlling for confounding factors by multivariate analysis. Glycemic control in general, and glycated hemoglobin in particular, is, of course, dependent on the level of knowledge. However, there are other factors on which this parameter depends, such as genetic, sociodemographic, psychological, and therapeutic factors [11, 22-25]. On the other hand, some areas of knowledge have no obvious link with glycemic control [7], either because of their purely theoretical aspect (such as knowledge of the etiology of diabetes), or because they only represent a particular situation in the life of the diabetic patient (such as what to do in sick days). The link between the level of knowledge and acute or chronic complications of diabetes was also not demonstrated in our study as well as in other studies [4]. This reflects that the occurrence of complications is dependent on other factors.

Concerning the other factors whose relation with the level of knowledge has been studied, older age was associated with lower levels of knowledge. This association was at the limit of significance in univariate analysis and was no longer present after multivariate analysis. However, many studies have shown that older age is associated with a lower level of knowledge [7, 8, 15]. This fact can be explained by the decrease with age of the initial knowledge provided [26]. This would also explain the relationship found by some studies between the duration of diabetes and the decline in the level of knowledge [5]. Gender, however, did not seem to influence the level of knowledge. Likewise, socioeconomic status no longer seems to be a confounding factor in its association with the level of knowledge since, after multivariate analysis, the relationship between these two parameters was no longer significant.

\section{Conclusions}

Improving diabetes knowledge of T1D patients may be helpful to achieve better glycemic control. In this study, level of education is one of the main factors independently associated with the level of knowledge of adult T1D patients. This should encourage healthcare professionals to better tailor their educational messages and resources to patients with low levels of education. Self-monitoring of blood glucose is also associated with the level of knowledge of adult T1D patients. This suggests that a better level of knowledge ensures better self-management of diabetes. However, the relationship with the quality of glycemic control remains uncertain.

\section{Conflict of interest}

None declared.

\section{REFERENCES}

1. Koontz MB, Cuttler L, Palmert MR, et al. Development and validation of a questionnaire to assess carbohydrate and insulindosing knowledge in youth with type 1 diabetes. Diabetes Care. 2010; 33(3): 457-462, doi: 10.2337/dc09-0390, indexed in Pubmed: 20007940.

2. Méndez FJ, Beléndez M. Effects of a behavioral intervention on treatment adherence and stress management in adolescents with IDDM. Diabetes Care. 1997; 20(9): 1370-1375, doi: 10.2337/ diacare.20.9.1370, indexed in Pubmed: 9283782 
3. Murphy HR, Rayman G, Skinner TC. Psycho-educational interventions for children and young people with type 1 diabetes. Diabet Med. 2006; 23(9): 935-943, doi: 10.1111/j.14645491.2006.01816.x, indexed in Pubmed: 16922699.

4. Abdul Wa, Amarrej HR, Al Au. Evaluation of the level of knowledge in type 1 diabetic patients \& their families. Iraqi J Comm Med. 2006; 19(2): 11

5. Sivagnanam G, Namasivayam K, Rajasekaran M, et al. A comparative study of the knowledge, beliefs, and practices of diabetic patients cared for at a teaching hospital (free service) and those cared for by private practitioners (paid service). Ann N Y Acad Sci. 2002; 958: 416-419, doi: 10.1111/j.1749-6632.2002.tb03016.x, indexed in Pubmed: 12021153.

6. Cuschieri S, Borg D, Pace S, et al. A review of diabetic patients' knowledge in a high prevalent European country - Malta. Malta Med J. 2016; 28: 36-45.

7. Speight J, Bradley C. The ADKnowl: identifying knowledge deficits in diabetes care. Diabet Med. 2001; 18(8): 626-633, doi: 10.1046/j.1464-5491.2001.00537.x, indexed in Pubmed: 11553199.

8. Fenwick EK, Xie J, Rees G, et al. Factors associated with knowledge of diabetes in patients with type 2 diabetes using the Diabetes Knowledge Test validated with Rasch analysis. PLoS One. 2013; 8(12): e80593, doi: 10.1371/journal.pone.0080593, indexed in Pubmed: 24312484.

9. Ozcelik F, Yiginer O, Arslan E, et al. Association between glycemic control and the level of knowledge and disease awareness in type 2 diabetic patients. Pol Arch Med Wewn. 2010; 120(10): 399-406, doi: 10.20452/pamw.979, indexed in Pubmed: 20980945.

10. Howells L, Wilson AC, Skinner TC, et al. A randomized control trial of the effect of negotiated telephone support on glycaemic control in young people with type 1 diabetes. Diabet Med. 2002; 19(8): 643-648, doi: 10.1046/j.1464-5491.2002.00791.x, indexed in Pubmed: 12147144.

11. Santos FR, Bernardo V, Gabbay MAL, et al. The impact of knowledge about diabetes, resilience and depression on glycemic control: a cross-sectional study among adolescents and young adults with type 1 diabetes. Diabetol Metab Syndr. 2013; 5(1): 55, doi: 10.1186/1758-5996-5-55, indexed in Pubmed: 24289093.

12. Smaldone A, Ganda OmP, McMurrich $S$, et al. Should group education classes be separated by type of diabetes? Diabetes Care. 2006; 29(7): 1656-1658, doi: 10.2337/dc06-0356, indexed in Pubmed: 16801595.

13. Rafique G, Azam SI, White F. Diabetes knowledge, beliefs and practices among people with diabetes attending a university hospital in Karachi, Pakistan. East Mediterr Health J. 2006; 12(5): 590-598, indexed in Pubmed: 17333798.
14. Tham KY, Ong JJ, Tan DK, et al. How much do diabetic patients know about diabetes mellitus and its complications? Ann Acad Med Singap. 2004; 33(4): 503-509, indexed in Pubmed: 15329765.

15. Jeppesen KM, Hull BP, Raines M, et al. A validation study of the spoken knowledge in low literacy in diabetes scale (SKILLD). J Gen Intern Med. 2012; 27(2): 207-212, doi: 10.1007/s11606011-1900-9, indexed in Pubmed: 22005940.

16. Marre M, Savanet JP. Ce qui permet à un diabétique type $1 \mathrm{~d}$ 'avoir sa glycémie sous controle. Diab Met. 2002; 28(2): 7-14.

17. Kanchana D, Parimalakrishnan S, Rakesh S. Assessment of diabetes knowledge using Diabetes Knowledge Questionnaire among people with diabetes type 2. Asian J Pharm Clin Res. Asian J Pharm Clin Res. 2015; 8(2): 254-256.

18. Brady B, Nies MA. Health-promoting lifestyles and exercise: a comparison of older African American women above and below poverty level. J Holist Nurs. 1999; 17(2): 197-207, doi: 10.1177/0 89801019901700207, indexed in Pubmed: 10633652.

19. American Association of Diabetes Educators. AADE position statement. Individualization of diabetes self-management education. Diabetes Educ. 2007; 33(1): 45-49, doi: 10.1177/0145721706298308, indexed in Pubmed: 17272792.

20. Sumner J, Baber C, Williams V. What do patients with type 1 diabetes know about hypoglycaemia? Practical Diabetes International. 2000; 17(6): 187-190, doi: 10.1002/1528-252x(200009)17:6<187::aidpdi74>3.0.co;2-i.

21. Hurley AC, Shea CA. Self-efficacy: strategy for enhancing diabetes self-care. Diabetes Educ. 1992; 18(2): 146-150, doi: 10.1177/01 4572179201800208, indexed in Pubmed: 1537242.

22. Snieder H, Sawtell PA, Ross L, et al. $\mathrm{HbA}(1 \mathrm{c})$ levels are genetically determined even in type 1 diabetes: evidence from healthy and diabetic twins. Diabetes. 2001; 50(12): 2858-2863, doi: 10.2337/ diabetes.50.12.2858, indexed in Pubmed: 11723071.

23. Devries JH, Snoek FJ, Heine RJ. Persistent poor glycaemic control in adult type 1 diabetes. A closer look at the problem. Diabet Med. 2004; 21(12): 1263-1268, doi: 10.1111/j.14645491.2004.01386.x, indexed in Pubmed: 15569126.

24. Tan SMK, Shafiee Z, Wu LL, et al. Factors associated with control of type I diabetes in Malaysian adolescents and young adults. Int J Psychiatry Med. 2005; 35(2): 123-136, doi: 10.2190/EQ71RMWV-6CEJ-1DGM, indexed in Pubmed: 16240970.

25. Yazidi M, Chihaoui M, Chaker F, et al. Factors predicting glycemic control in type 1 diabetic patient. Open Medicine Journal. 2016; 3(1): 153-158, doi: 10.2174/1874220301603010153.

26. Bulikaitè V, Drukteinienè S. Assessment of skills and knowledge about the disease among teenagers with type 1 diabetes mellitus. NERP. 2013; 3(5): 175-181. 